\title{
Und wieder ist ein Jahr vorbei - oder auch fast schon zwei...
}

Johannes Rux*

Dieses Heft widmet sich fast vollständig dem Thema der Kodifikation. Vier große Abhandlungen beleuchten das Thema aus den verschiedenen Perspektiven der einzelnen Rechtsgebiete. Leider erscheint das Heft mit einer gehörigen Verspätung. Dafür möchten wir uns bei unseren Leserinnen und Lesern entschuldigen. Aber wir sind überzeugt, dass sich das Warten gelohnt hat.

Wie in jedem Jahr danken wir an dieser Stelle denjenigen, die uns in den letzten Monaten als Peers mit ihren Gutachten sehr geholfen haben. Unser Dank gilt: Christian Armbrüster (Berlin), Marietta Auer (München), Brun-Otto Bryde (Gießen), Jochen Bung (Passau), Martin Burgi (München), Horst Dreier (Würzburg), Wolfgang Durner (Bonn), Eberhard Eichenhofer (Jena), Jörg Eisele (Konstanz), Albin Eser (Freiburg), Elke Gurlit (Mainz), Hans Hanau (Hamburg), Armin Hatje (Hamburg), Stefan Huster (Bochum), Gerhard Igl (Kiel), Lorenz Kähler (Bremen), Christian Katzenmeier (Köln), Hans Kudlich (Erlangen), Silke-Ruth Laskowski (Kassel), Michele Luminati (Luzern), Ralf Poscher (Freiburg), Matthias Rohe (Erlangen), Ute Sacksofsky (Frankfurt), Arno Scherzberg (Erfurt), Martin Schmidt-Kessel (Bayreuth), Margarethe Schuler-Harms (Hamburg), Hans Schulte-Nölke (Osnabrück), Andreas Thier (Zürich), Thomas Vesting (Hamburg), Andreas von Arnauld (Münster), MarcPhilippe Weller (Freiburg).

Natürlich wollen wir immer noch besser werden - und laden Sie ein, sich mit eigenen Beiträgen am fachgebietsübergreifenden Diskurs zu beteiligen und dazu beizutragen, die Grenzen zwischen den juristischen Disziplinen zumindest durchlässiger zu machen.

Die Kontaktdaten der Schriftleitung finden Sie unten auf Seite 2 des Heftes. Hinweise für die Manuskriptgestaltung sind auf der Internet-Seite www.rechtswissenschaft.nomos.de abrufbar.

* Prof. Dr. Johannes Rux lehrt Öffentliches Recht an der Eberhard-Karls-Universität Tübingen und ist Schriftleiter der RECHTSWISSENSCHAFT. 